\title{
Biliary peritonitis due to liver cyst rupture in autosomal dominant polycystic kidney disease
}

\author{
Hiroshi Matsuo ${ }^{1,2}$, Kan Katayama ${ }^{2 *}$, Aoi Hayasaki ${ }^{3}$, Yusuke lizawa $^{3}$, Mayumi Endo $^{1,2}$, Tomohiro Murata ${ }^{2}$,
} Shugo Mizuno ${ }^{3}$ and Kaoru Dohi

\begin{abstract}
Background: Autosomal dominant polycystic kidney disease (ADPKD) is the most frequent genetic kidney disease and polycystic liver disease is its major extrarenal manifestation, however biliary peritonitis due to a liver cyst rupture is extremely rare.

Case presentation: The patient was a 71-year-old Japanese woman who was diagnosed with ADPKD 3 years previously and developed right abdominal pain suddenly 1 month previously. As abdominal computed tomography (CT) showed a ruptured liver cyst in the right lobe, she was admitted to our hospital. Her symptoms improved with conservative management and she was discharged from the hospital after 1 week. Although she was asymptomatic for a while, she noticed abdominal distension and general malaise at 1 month after hospital discharge. Since abdominal CT showed massive ascites, she was admitted to our hospital again. A physical examination revealed abdominal distention without tenderness. Her serum creatinine, alkaline phosphatase, $\gamma$-glutamyl transpeptidase, total bilirubin, and CA19-9 were elevated. Abdominal paracentesis revealed amber transparent ascites and the bilirubin and CA19-9 concentrations were high. She was diagnosed with biliary peritonitis due to a ruptured liver cyst. Hemodialysis treatment was initiated with drainage of the ascites. The outflow of the ascites was no tendency to decrease and drip infusion cholangiography (DIC)-CT revealed a communication between the ruptured cyst and an intrahepatic bile duct. On day 31, she was transferred to a university hospital and abdominal surgery was performed. After removing the necrotic roof of the ruptured cyst on the right liver lobe, the orifice of the bile leakage was sutured. Cholecystectomy was performed and cholangiography showed no stones in the common bile duct. Abdominal CT one month after the operation showed no recurrence of ascites and she was discharged on day 49 . Hemodialysis treatment was discontinued immediately after discharge because urine volume increased and her creatinine level decreased. There has been no recurrence of ascites since then.
\end{abstract}

Conclusions: While rare, biliary peritonitis can occur in association with the rupture of a liver cyst in ADPKD patients due to communication between the cyst and the intrahepatic bile duct, and DIC-CT should be recommended when biliary cyst rupture is suspected.

Keywords: Autosomal dominant polycystic kidney disease, Biliary peritonitis, Liver cyst rupture

*Correspondence: katayamk@clin.medic.mie-u.ac.jp

2 Department of Cardiology and Nephrology, Mie University Graduate

School of Medicine, 2-174 Edobashi, Tsu, Mie 514-8507, Japan

Full list of author information is available at the end of the article

\section{Background}

Autosomal dominant polycystic kidney disease (ADPKD) is the most frequent genetic kidney disease; the prevalence is 1 in 500-1000 [1]. There are two major causative 
genes: PKD1 and PKD2. The onset of end-stage kidney disease due to multiple cysts in both kidneys is much slower in patients with PKD2 mutation than in those with PKD1 mutation [2].

Extrarenal manifestations, include polycystic liver disease (PLD), intracranial aneurysm, mitral valve prolapse, and colon diverticulosis [3]. While PLD due to ADPKD is basically asymptomatic, acute symptomatic complications of single liver cysts can occur, including cyst infection, hemorrhage, torsion, or rupture [4]. We experienced a rare case of biliary peritonitis due to the rupture of a liver cyst in a patient with ADPKD, which was successfully treated with abdominal surgery.

\section{Case presentation}

The patient was a 71-year-old Japanese woman who was diagnosed with chronic kidney disease due to ADPKD 3 years previously. She received surgical clipping for an unruptured aneurysm of the right middle cerebral artery 4 years earlier. She did not drink alcohol and was a nonsmoker. She was taking amlodipine for hypertension, topiroxostat for hyperuricemia, and ferric citrate hydrate for hyperphosphatemia. Continuous erythropoietin receptor activator was administered subcutaneously for renal anemia once a month. She was followed up in an outpatient clinic and suddenly developed right abdominal pain one month previously. As abdominal computed tomography $(\mathrm{CT})$ showed a ruptured liver cyst in the right lobe, which had not been observed seven months previously (Fig. 1a, b), she was admitted to our hospital. While her white blood cell count was within the normal range at $5900 / \mu \mathrm{L}, \mathrm{C}$-reactive protein was a little high at $0.4 \mathrm{mg} / \mathrm{dL}$. She was treated with bed rest and intravenous cefmetazole administration ( $1 \mathrm{~g} /$ day) on suspicion of cyst infection. Oral acetaminophen $500 \mathrm{mg}$ was occasionally used when needed, and her symptoms improved with conservative management and she was discharged from the hospital after one week. Although she was asymptomatic for a while, she noticed bilateral leg edema, abdominal distension, and general malaise at one month after hospital discharge. Since abdominal CT showed massive ascites (Fig. 1c), she was admitted to our hospital again.

On admission, her height was $166 \mathrm{~cm}$, and her body weight was $62 \mathrm{~kg}$. A physical examination revealed the following: body temperature, $37.2{ }^{\circ} \mathrm{C}$; blood pressure, 114/73 mmHg; pulse, 94 beats/min; and oxygen rate, $97 \%$ on room air. Abdominal distention was observed without tenderness, and pitting edema was observed in the bilateral legs. The laboratory data are shown in Table 1 . Her serum creatinine, alkaline phosphatase, $\gamma$-glutamyl transpeptidase, total bilirubin, and CA19-9 were $5.2 \mathrm{mg} /$ $\mathrm{dL}, 1992 \mathrm{U} / \mathrm{L}, 355 \mathrm{U} / \mathrm{L}, 3.1 \mathrm{mg} / \mathrm{dL}$, and $5006 \mathrm{U} / \mathrm{mL}$, respectively.
Abdominal paracentesis revealed amber transparent ascites (Fig. 1d) and the bilirubin and CA19-9 concentrations were high at $13.3 \mathrm{mg} / \mathrm{dL}$ and $244,700 \mathrm{U} / \mathrm{mL}$, respectively. Ascites culturing and cytology were negative. She was diagnosed with biliary peritonitis due to a ruptured liver cyst. Hemodialysis was initiated due to the exacerbation of chronic kidney disease. As conservative treatment did not improve her symptom, drainage of the ascites was initiated from day 11 . The outflow of the ascites was $1000 \mathrm{~mL}$ per day and there was no tendency to decrease. Drip infusion cholangiography (DIC)-CT revealed that the ruptured cyst in the right posterior segment $\mathrm{S} 6$ of the liver was enhanced with contrast medium (Fig. 1e). Three-dimensional views of DIC-CT showed communication between the ruptured cyst and an intrahepatic bile duct (Fig. 1f, g). Because of her markedly high CA19-9 levels in serum and ascites, upper endoscopy and repeated ascites cytology were required to rule out cancer. On day 24, oral cefditoren pivoxil $(100 \mathrm{mg} /$ day) was initiated because her $C$-reactive protein was still high at $7.4 \mathrm{mg} / \mathrm{dL}$. On day 31 , she was transferred to a university hospital, and the ascites culture on the same day was found to be positive for Enterococcus faecalis. Abdominal surgery was performed. The surface of the liver became yellowish due to bile leakage and a ruptured necrotic cyst was observed on the right liver lobe (Fig. $1 \mathrm{~h}$ ). After removing the necrotic roof, the orifice that was the source of the bile leakage was identified and sutured (Fig. 1i). As gallbladder stones had been pointed out, cholecystectomy was performed at the same time. Cholangiography showed no stones in the common bile duct. Intravenous flomoxef sodium $(0.5 \mathrm{~g} / \mathrm{day})$ was initiated for 3 consecutive days, followed by intravenous sulbactam/ampicillin ( $1.5 \mathrm{~g} /$ day) for 12 days. Abdominal CT one month after the operation showed no recurrence of ascites and she was discharged on day 49 . Hemodialysis treatment was discontinued immediately after discharge because her urine volume had increased and her creatinine level had decreased to $3.8 \mathrm{mg} / \mathrm{dL}$. There has been no recurrence of ascites since then.

\section{Discussion and conclusions}

We experienced a rare case of biliary peritonitis due to a ruptured liver cyst in an ADPKD patient. While the first episode of liver cyst rupture improved with conservative management, the second episode caused massive ascites. Abdominal paracentesis was useful for confirming the diagnosis of biliary peritonitis, which was successfully treated with abdominal surgery.

Liver cysts are asymptomatic in most settings and liver cyst rupture is rare [5]. Marion et al. reviewed 11 cases of hemorrhagic liver cyst rupture, of which 4 cases involved patients with ADPKD [6]. Three of the four cases had a 

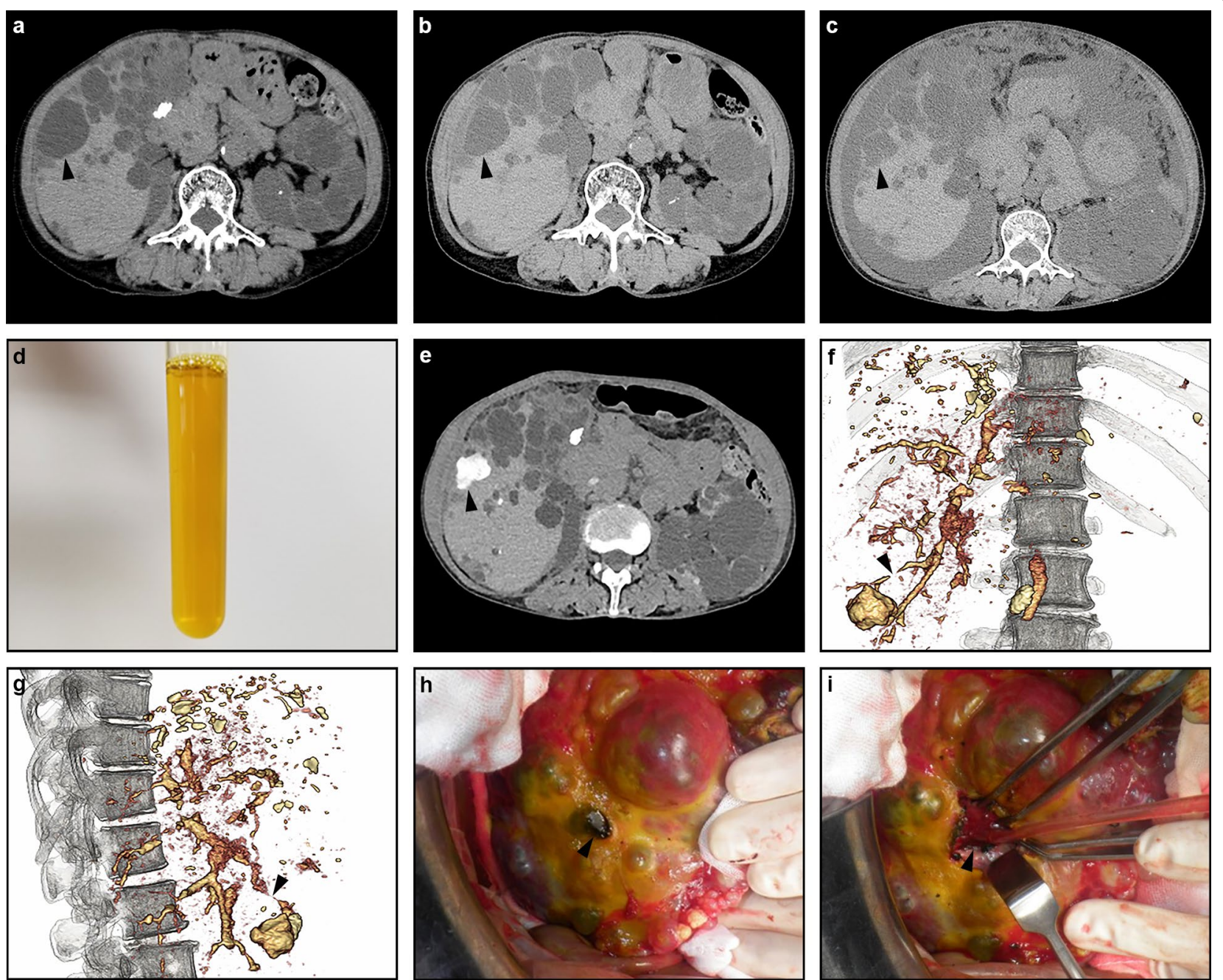

Fig. 1 a Abdominal computed tomography (CT) 7 months previously showed gallbladder stones and multiple cysts in the liver and the left kidney. b Abdominal CT at the time of the first admission showed a ruptured liver cyst in the right lobe (arrowhead) while it was not observed seven months previously (arrowhead). c Abdominal CT on the second admission showed that the ruptured cyst (arrowhead) was reduced with massive ascites. $\mathbf{d}$ Abdominal paracentesis revealed amber transparent ascites. e Drip infusion cholangiography (DIC)-CT revealed that the ruptured cyst in the right posterior segment $\mathbf{S} 6$ of the liver was enhanced with contrast medium (arrowhead). $\mathbf{f}, \mathbf{g}$ Three-dimensional (3D) views of DIC-CT showed communication between the ruptured cyst and the intrahepatic bile duct (arrowheads, $\mathbf{f}$; front view, $\mathbf{g}$; lateral view). $\mathbf{h}$ The surface of the liver became yellowish due to bile leakage and a ruptured necrotic cyst (arrowhead) was observed on the right liver lobe. i After removing the necrotic roof, the orifice (arrowhead) that was the source of the bile leakage was identified

hemodynamic impact and two of the four cases were fatal [7-10]. There was also a case of fatal liver cyst rupture in a patient with ADPKD due to trauma [11]. Another report showed that acute abdomen and ascites due to liver cyst rupture led to the diagnosis of ADPKD [12]. However, to the best of our knowledge, there have been no reports on biliary peritonitis due to spontaneous liver cyst rupture in a patient with ADPKD.

While ruptures of the gallbladder, common bile duct [13], extrahepatic bile duct [14], and intrahepatic bile duct $[15,16]$ are considered to be causes of spontaneous biliary peritonitis, the development of peritonitis in association with the rupture of a liver cyst communicating with a biliary tract is rare. Threedimensional DIC-CT showed dilatations of the distal intrahepatic bile ducts near the liver surface, suggesting partially increased internal pressure of the bile duct with biliary stasis. There have been reported cases involving a liver cyst with biliary communication and both cases were treated by laparoscopic deroofing before rupture $[17,18]$. The cystobiliary communication was found by percutaneous transhepatic drainage 
Table 1 Laboratory data on admission

\begin{tabular}{|c|c|c|}
\hline Parameter & Patient value & Reference \\
\hline \multicolumn{3}{|l|}{ Urinalysis } \\
\hline Protein & $(-)$ & $(-)$ \\
\hline Occult blood & $1+$ & $<(+-)$ \\
\hline \multicolumn{3}{|l|}{ Hematology } \\
\hline White blood cells $(/ \mu \mathrm{L})$ & 4600 & $4000-9000$ \\
\hline Red blood cells $\left(\times 10^{4} / \mu \mathrm{L}\right)$ & 332 & $380-480$ \\
\hline Hemoglobin (g/dL) & 10.5 & $12.0-16.0$ \\
\hline Hematocrit (\%) & 32.1 & $34.0-42.0$ \\
\hline Platelets $\left(\times 10^{4} / \mu \mathrm{L}\right)$ & 12.6 & $12.0-40.0$ \\
\hline \multicolumn{3}{|l|}{ Coagulation } \\
\hline Activated partial thromboplastin time (s) & 29 & $21-36$ \\
\hline $\begin{array}{l}\text { Prothrombin time international normalized } \\
\text { ratio }\end{array}$ & 1.06 & $0.88-1.08$ \\
\hline Fibrinogen (mg/dL) & 568 & $200-400$ \\
\hline \multicolumn{3}{|l|}{ Blood chemistry } \\
\hline Total protein (g/dL) & 6.7 & $6.5-8.5$ \\
\hline Albumin (g/dL) & 2.8 & $4.1-5.3$ \\
\hline Blood urea nitrogen (mg/dL) & 47.1 & $9.0-20.0$ \\
\hline Creatinine (mg/dL) & 5.21 & $0.50-1.10$ \\
\hline Aspartate aminotransferase $(\mathrm{U} / \mathrm{L})$ & 27 & $5-40$ \\
\hline Alanine aminotransferase $(U / L)$ & 12 & $4-44$ \\
\hline Lactic dehydrogenation enzyme (U/L) & 182 & $110-225$ \\
\hline Alkaline phosphatase (U/L) & 1992 & $120-340$ \\
\hline Y-Glutamyl transpeptidase (U/L) & 355 & $0-60$ \\
\hline Total bilirubin (mg/dL) & 3.1 & $0.2-1.3$ \\
\hline Direct bilirubin (mg/dL) & 2.6 & $0.0-0.5$ \\
\hline Uric acid (mg/dL) & 6 & $2.5-7.0$ \\
\hline Natrium (mmol/L) & 135 & $136-148$ \\
\hline Potassium (mmol/L) & 4 & $3.5-5.0$ \\
\hline Chlorine (mmol/L) & 99 & $98-110$ \\
\hline Calcium (mg/dL) & 8.2 & $8.5-10.5$ \\
\hline Inorganic phosphate (mg/dL) & 4.3 & $2.5-4.5$ \\
\hline C-reactive protein (mg/dL) & 4.97 & $<0.30$ \\
\hline Total cholesterol (mg/dL) & 129 & $150-219$ \\
\hline Triglyceride (mg/dL) & 51 & $50-150$ \\
\hline \multicolumn{3}{|l|}{ Serological tests } \\
\hline HBs antigen & $(-)$ & $(-)$ \\
\hline Anti-HCV antibody & $(-)$ & $(-)$ \\
\hline CA19-9 (U/mL) & 5006 & $0-37$ \\
\hline \multicolumn{3}{|l|}{ Ascites } \\
\hline Total protein (g/dL) & 2.9 & \\
\hline Albumin (g/dL) & 1.3 & \\
\hline Total bilirubin (mg/dL) & 13.3 & \\
\hline Adenosine deaminase $(\mathrm{U} / \mathrm{L})$ & 7.9 & $5-20$ \\
\hline CA19-9 (U/mL) & 244,700 & \\
\hline
\end{tabular}

of the cyst before the operation in one report [17], while it was recognized during laparoscopic deroofing in the other report [18]. The difficulty in finding cystobiliary communication might be attributed to the high intracystic pressure [18]. While the first ascites culture was negative in the present case, the existence of bile in the abdominal cavity increased the risk of secondary bacterial infection [19]. Indeed, the second ascites culture before operation was positive for Enterococcus faecalis. Abdominal surgery instead of laparoscopy, or non-operative approaches, such as percutaneous transhepatic biliary drainage, was chosen in the present case for several reasons. First, massive leakage of the bile was suspected because the amount of biliary ascites exceeded $1000 \mathrm{~mL}$ per day. Second, the ascites culture was positive for E. faecalis before operation. Third, the ruptured cyst was a small cyst located among multiple cysts, so there was a possibility that the orifice would not be visible under laparoscopy. Abdominal surgery to close the orifice that was the source of the bile leakage in the ruptured cyst achieved a favorable outcome without severe bacterial infection in the present case. We do not think the present case was indicated for liver transplantation, as closure of the orifice of the bile leakage was successful; however, liver transplantation may be a viable option in cases of repetitive biliary peritonitis due to many hepatobiliary communications after controlling the infection and taking into account the patient's age and other comorbidities.

In conclusion, while rare, biliary peritonitis can occur in association with the rupture of a liver cyst in ADPKD patients due to communication between the cyst and the intrahepatic bile duct, and DIC-CT should be recommended when biliary cyst rupture is suspected.

\section{Abbreviations}

ADPKD: Autosomal dominant polycystic kidney disease; CT: Computed tomography; DIC: Drip infusion cholecystocholangiography; PLD: Polycystic liver disease.

\section{Acknowledgements}

Not applicable.

\section{Authors' contributions}

$\mathrm{HM}, \mathrm{KK}, \mathrm{AH}, \mathrm{YI}, \mathrm{ME}$, and TM participated in the acquisition of clinical data. HM, KK, SM, and TM carried out analysis of patient's clinical course and data interpretation. HM and KK wrote a draft of the manuscript and $\mathrm{AH}, \mathrm{YI}, \mathrm{ME}, \mathrm{TM}, \mathrm{SM}$, and $\mathrm{KD}$ revised it critically. All authors read and approved the final manuscript.

Funding

Not applicable.

\section{Availability of data and materials}

The datasets used and/or analyzed during the current study are available from the corresponding author on reasonable request.

\section{Declarations}

Ethics approval and consent to participate Not applicable. 


\section{Consent for publication}

Written informed consent was obtained from the patient for the publication of this Case report. A copy of the written consent is available for review by the editor of this journal.

\section{Competing interests}

The authors declare that they have no competing interests.

\section{Author details}

${ }^{1}$ Kidney Center, Suzuka Kaisei Hospital, Suzuka, Japan. ${ }^{2}$ Department of Cardiology and Nephrology, Mie University Graduate School of Medicine, 2-174 Edobashi, Tsu, Mie 514-8507, Japan. ${ }^{3}$ Department of Hepatobiliary Pancreatic and Transplant Surgery, Mie University Graduate School of Medicine, Tsu, Japan.

Received: 30 March 2021 Accepted: 14 June 2021

Published online: 24 June 2021

\section{References}

1. Simons $M$, Walz G. Polycystic kidney disease: cell division without a c(l)ue? Kidney Int. 2006;70:854-64.

2. Hateboer N, v Dijk MA, Bogdanova N, Coto E, Saggar-Malik AK, San Millan $J$, et al. Comparison of phenotypes of polycystic kidney disease types 1 and 2. European PKD1-PKD2 Study Group. Lancet. 1999:353:103-7.

3. Torres VE, Harris PC, Pirson Y. Autosomal dominant polycystic kidney disease. Lancet. 2007;369:1287-301.

4. Chauveau D, Fakhouri F, Grünfeld JP. Liver involvement in autosomaldominant polycystic kidney disease: therapeutic dilemma. J Am Soc Nephrol. 2000;11:1767-75.

5. van Aerts RMM, van de Laarschot LFM, Banales JM, Drenth JPH. Clinical management of polycystic liver disease. J Hepatol. 2018;68:827-37.

6. Marion Y, Brevartt C, Plard L, Chiche L. Hemorrhagic liver cyst rupture: an unusual life-threatening complication of hepatic cyst and literature review. Ann Hepatol. 2013;12:336-9.

7. Fidas-Kamini A, Busuttil A. Fatal intraperitoneal haemorrhage of hepatic origin. Postgrad Med J. 1986;62:1097-100.
8. Rutecki GW, Asfoura JY, Whittier FC. Autosomal dominant polycystic liver disease as an etiology for hemoperitoneum during CCPD. Perit Dial Int. 1995:15:367-9.

9. Chung TK, Chen KS, Yen CL, Chen HY, Cherng WJ, Fang KM. Acute abdomen in a haemodialysed patient with polycystic kidney disease-rupture of a massive liver cyst. Nephrol Dial Transplant. 1998;13:1840-2.

10. Carels RA, van Bommel EF. Ruptured giant liver cyst: a rare cause of acute abdomen in a haemodialysis patient with autosomal dominant polycystic kidney disease. Neth J Med. 2002;60:363-5.

11. Tong F, Liang Y, Zhang L, Li W, Chen P, Duan Y, et al. Fatal liver cyst rupture in polycystic liver disease complicated with autosomal dominant polycystic kidney disease: a case report. Forensic Sci Int. 2016;262:e5-8.

12. Chaudhary S, Qian Q. Acute abdomen and ascites as presenting features of autosomal dominant polycystic kidney disease. World J Hepatol. 2012;4:394-8.

13. Kerstein MD, McSwain NE. Spontaneous rupture of the common bile duct. Am J Gastroenterol. 1985;80:469-71.

14. Chardot C, Iskandarani F, De Dreuzy O, Duquesne B, Pariente D, Bernard O, et al. Spontaneous perforation of the biliary tract in infancy: a series of 11 cases. Eur J Pediatr Surg. 1996:6:341-6.

15. Chalasani N, Nguyen CC, Gitlin N. Spontaneous rupture of a bile duct and its endoscopic management in a patient with Caroli's syndrome. Am J Gastroenterol. 1997;92:1062-3.

16. Lochan $\mathrm{R}$, Joypaul BV. Bile peritonitis due to intra-hepatic bile duct rupture. World J Gastroenterol. 2005:11:6728-9.

17. Masatsugu T, Shimizu S, Noshiro H, Mizumoto K, Yamaguchi K, Chijiiwa $\mathrm{K}$, et al. Liver cyst with biliary communication successfully treated with laparoscopic deroofing: a case report. JSLS. 2003;7:249-52.

18. Yamada T, Furukawa K, Yokoi K, Mamada Y, Kanazawa Y, Tajiri T. Liver cyst with biliary communication successfully treated with laparoscopic deroofing: a case report. J Nippon Med Sch. 2009;76:103-8.

19. Andersson R, Tranberg KG, Bengmark $S$. Roles of bile and bacteria in biliary peritonitis. Br J Surg. 1990;77:36-9.

\section{Publisher's Note}

Springer Nature remains neutral with regard to jurisdictional claims in published maps and institutional affiliations.
Ready to submit your research? Choose BMC and benefit from:

- fast, convenient online submission

- thorough peer review by experienced researchers in your field

- rapid publication on acceptance

- support for research data, including large and complex data types

- gold Open Access which fosters wider collaboration and increased citations

- maximum visibility for your research: over 100M website views per year

At BMC, research is always in progress.

Learn more biomedcentral.com/submissions 